\title{
Preface: Nuclear Structure and Dynamics IV - NSD2019
}

\author{
Giacomo de Angelis ${ }^{1, *}$ and Lorenzo Corradi ${ }^{1, *}$ \\ ${ }^{1}$ INFN - Laboratori Nazionali di Legnaro, Italy
}

The fourth International Conference on Nuclear Structure and Dynamics NSD2019 was held on May 13-17, 2019 at "Centro Culturale Don Orione Artigianelli" in Venice within the exciting context of the $58^{\text {th }}$ International Art Exhibition. The Center is a large complex, built as a monastery and later used as an orphanage and vocational school. The entire building develops around three cloisters of the $18^{\text {th }}$ century and one cloister of the $15^{\text {th }}$ century near the Church of Santa Maria della Visitazione. It provided a beautiful and peaceful atmosphere for stimulating discussions. The conference, organized by the INFN Laboratori Nazionali di Legnaro and the Physics and Astronomy Department of the Padova University, was a follow-up of the three previous conferences held first in 2009 in Dubrovnik (Croatia), continued in 2012 in Opatija (Croatia) and last in 2015 in Portoroz (Slovenia), and belongs to a series of conferences devoted to the most recent experimental and theoretical advances in the field of nuclear structure and reactions. NSD2019 maintained this tradition, promoting exchange of ideas and collaboration among researchers, post-docs and graduate students with experimental, theoretical and phenomenological background. According to the tradition of this series of conferences, the focus was on topics covering most of the research areas in low energy nuclear physics:

- Nuclear structure and reactions far from stability

- Collective phenomena and symmetries

- Dynamics and thermodynamics of light and heavy nuclei

- Sub and near barrier reactions

- Fusion and Fission dynamics

- Ab initio calculations, cluster models and shell model

- Nuclear energy density functionals

- Nuclear astrophysics

- Fundamental interactions.

About 150 physicists from all over the world attended the conference. The opening ceremony took place in the morning of May $13^{\text {th }}$. After a short introduction of the conference chairs (Giacomo de Angelis and Lorenzo Corradi), the participants were welcomed by the director of the Legnaro National
Laboratory (Diego Bettoni). 30 invited speakers and 60 regular talks, some of which scheduled in parallel sessions, covered a wide range of topics. More than 30 posters were presented at the poster session. The three best voted ones were discussed as short oral presentations. We paid tribute to our colleague Graziano Fortuna, former director of the Legnaro National Laboratory, who very recently passed away.

Important new results were outlined in the various subfields, spanning the whole range from neutrondeficient to neutron-rich nuclei and from light to heavy ions. The talks, covering state-of-art experimental methods and theoretical developments, had as main reference the tight interconnection between structure and dynamics, enhancing attendees to an active participation and prompting lively discussions.

We sincerely appreciated the cooperative contribution of all members of the Organizing Committee: Enrico Fioretto, Tommaso Marchi, Marco Mazzocco, Daniele Mengoni, José Javier Valiente Dobón. Special thanks to our secretaries Anna D'Este and Adriana Schiavon for their skill and effort in following all aspects of the conference and making the event so successful.

We wish to warmly thank the members of the International Advisory Committee for helping us in the selection of speakers and precious suggestions: Rob Bark, Piotr Bednarczyck, Klaus Blaum, Francesco Cappuzzello, Michael Patten Carpenter, Giovanni Casini, Wilton Catford, Bo Cederwall, José Luis Egido, Angela Gargano, Paul Greenless, Alessandra Guglielmetti, Joseph Hamilton, Denis Lacroix, Chengjian Lin, Nicolae Marginean, Matko Milin, Petr Navratil, Gerda Neyens, Katsuhisa Nishio, Robert Page, Norbert Pietralla, Riccardo Raabe, Berta Rubio, Hendrik Shatz, Sergey Sidorchuck, Cedric Simenel, Ram Prakash Singh, Olivier Sorlin, Siem Sunniva, Tomohiro Uesaka, Andrea Vitturi, Dario Vretenar, Yu Hu Zhang.

We are finally very thankful to Dario Vretenar for having accepted the challenging task to held the Summary talk. His presentation underlined some of the major achievements of modern nuclear physics research presented during the conference.

\footnotetext{
* Corresponding author: deangelis@lnl.infn.it, corradi@lnl.infn.it
} 